\title{
Reliability and validity of the multi-point method and the 2- point method's variations of estimating the one-repetition maximum for deadlift and back squat exercises
}

\author{
Onat Çetin $^{1}$, Zeki Akyildiz ${ }^{2}$, Barbaros Demirtaş ${ }^{3}$, Yılmaz Sungur ${ }^{4,5}$, Filipe Manuel Clemente ${ }^{6}$, Florin Cazan ${ }^{7}$, Luca \\ P Ardigò ${ }^{\text {Corresp. } 8}$ \\ ${ }^{1}$ Faculty of Sports Sciences, Department of Coaching Education, Yalova University, Yalova, Turkey \\ 2 Faculty of Sports Sciences, Gazi University, Ankara, Turkey \\ 3 Movement and Training Sciences Department, Sports Sciences Faculty, Sakarya Applied Sciences University, Sakarya, Turkey \\ 4 Department of Movement and Training Science, Faculty of Sports Sciences, Akdeniz University, Antalya, Turkey \\ 5 Sports Medicine and Athletic Performance Department, Gloria Sports Arena, Antalya, Turkey \\ 6 Escola Superior Desporto e Lazer, Instituto Politécnico de Viana do Castelo, Viana do Castelo, Portugal \\ 7 Faculty of Physical Education and Sport, Ovidius University of Constanta, Constanta, Romania \\ 8 Department of Neurosciences, Biomedicine and Movement Sciences, University of Verona, Verona, Italy \\ Corresponding Author: Luca P Ardigò \\ Email address: luca.ardigo@univr.it
}

This study aimed at examining the concurrent validity and reliability of the multi-point method and the two-point method's variations for estimating the one-repetition maximum (1RM) in the deadlift and squat exercises and to determine the accuracy of which optimal two loads can be used for the 2-point method protocol. Thirteen resistance-trained men performed 6 sessions that consisted of 2 incremental loading tests (multi-point method: 20-40-60-80-90\% and two-point method variations: 40-60\%, 40-80\%, 40-90\%,60-80\%, $60-90 \%)$ followed by 1RM tests. Both the multi-point method and the two-point method load variations showed reliable results for $1 \mathrm{RM}$ estimation $(\mathrm{CV}<10 \%)$ squat and deadlift exercises. Session-session reliability was found to be low in deadlift (ICC: $0.171-0.335$ ) and squat exercises (ICC: $0.235-0.479$ ) of $40-60 \%$ and $60-80 \%$ in two-point methods. Deadlift (ICC: $0.815-0.996)$ and squat (ICC: $0.817-0.988$ ) had high session-to-session reliability in all other methods. Regarding the validity of deadlift exercise, the multipoint method $\left(R^{2}=0.864\right)$ and 2 variations of the two-point method $\left(R^{2}=0.816\right.$ for $40-80 \%, R^{2}=0.732$ for $60-80 \%)$ showed very large correlations, whereas other 2 variations of the two-point method $\left(R^{2}=0.945\right.$ for $40-90 \%, R^{2}=0.914$ for $60-90 \%$ ) showed almost perfect correlations with the actual 1RM. Regarding the validity of squat exercise, the multi-point method $\left(R^{2}=0.773\right)$ and 2 variations of the two-point method $\left(R^{2}=0.0847\right.$ for $60-80 \% \%, R^{2}=0.705$ for $40-90 \%$ ) showed very large correlations, whereas $40-60 \%$ variation showed almost 
perfect correlation $\left(R^{2}=0.962\right)$ with the actual $1 R M$. In conclusion, whereas both the multipoint method and the two-point method load variations showed reliable results, the multiple-point method and most of the two-point methods' load variations examined in this research provided an accurate (from large-moderate to perfect) estimate of the 1RM. Therefore, we recommend using the multi-point method and especially the two-point methods variations including higher relative loads to estimate 1RM. 
1 Reliability and validity of the multi-point method and 2 the 2-point method's variations of estimating the one3 repetition maximum for deadlift and back squat 4 exercises

5

6

Onat Çetin ${ }^{1}$, Zeki Akyildiz ${ }^{2}$, Barbaros Demirtaş ${ }^{3}$, Yılmaz Sungur ${ }^{4,5}$, Filipe Manuel Clemente ${ }^{6}$, Florin Cazan ${ }^{7}$, Luca Paolo Ardigò ${ }^{8}$

${ }^{1}$ Department of Coaching Education, Faculty of Sports Sciences, Yalova University, Yalova, Turkey

${ }^{2}$ Faculty of Sports Sciences, Gazi University, Ankara, Turkey

${ }^{3}$ Movement and Training Sciences Department, Sports Sciences Faculty, Sakarya Applied Sciences University, Sakarya, Turkey

${ }^{4}$ Department of Movement and Training Science, Faculty of Sports Sciences, Akdeniz University, Antalya, Turkey

${ }^{5}$ Sports Medicine and Athletic Performance Department, Gloria Sports Arena, Antalya, Turkey

${ }^{6}$ Escola Superior Desporto e Lazer, Instituto Politécnico de Viana do Castelo, Viana do Castelo, Portugal

${ }^{7}$ Faculty of Physical Education and Sport, Ovidius University of Constanta, Constanta, Romania

${ }^{8}$ Department of Neurosciences, Biomedicine and Movement Sciences, School of Exercise and Sport Science, University of Verona, Verona, Italy

Corresponding Author:

Luca Paolo Ardigò ${ }^{8}$

Via Felice Casorati, 43, Verona, 37131, Italy

Email address: luca.ardigo@univr.it

\section{Abstract}

This study aimed at examining the concurrent validity and reliability of the multi-point method and the two-point method's variations for estimating the one-repetition maximum (1RM) in the deadlift and squat exercises and to determine the accuracy of which optimal two loads can be used for the 2-point method protocol. Thirteen resistance-trained men performed 6 sessions that consisted of 2 incremental loading tests (multi-point method: 20-40-60-80-90\% and two-point method variations: $40-60 \%, 40-80 \%, 40-90 \%, 60-80 \%, 60-90 \%$ ) followed by 1 RM tests.

Both the multi-point method and the two-point method load variations showed reliable results for 1RM estimation $(\mathrm{CV}<10 \%)$ squat and deadlift exercises. Session-session reliability was found to be low in deadlift (ICC: $0.171-0.335$ ) and squat exercises (ICC: $0.235-0.479)$ of $40-60 \%$ and 60 - 
80\% in two-point methods. Deadlift (ICC: 0.815-0.996) and squat (ICC: 0.817-0.988) had high session-to-session reliability in all other methods. Regarding the validity of deadlift exercise, the multipoint method $\left(\mathrm{R}^{2}=0.864\right)$ and 2 variations of the two-point method $\left(\mathrm{R}^{2}=0.816\right.$ for $40-80 \%$, $\mathrm{R}^{2}=0.732$ for $60-80 \%$ ) showed very large correlations, whereas other 2 variations of the twopoint method $\left(\mathrm{R}^{2}=0.945\right.$ for $40-90 \%, \mathrm{R}^{2}=0.914$ for $\left.60-90 \%\right)$ showed almost perfect correlations with the actual 1RM. Regarding the validity of squat exercise, the multi-point method $\left(\mathrm{R}^{2}=0.773\right)$ and 2 variations of the two-point method $\left(\mathrm{R}^{2}=0.0847\right.$ for $60-80 \% \%, \mathrm{R}^{2}=0.705$ for 40 $90 \%$ ) showed very large correlations, whereas $40-60 \%$ variation showed almost perfect correlation $\left(\mathrm{R}^{2}=0.962\right)$ with the actual $1 \mathrm{RM}$. In conclusion, whereas both the multi-point method and the two-point method load variations showed reliable results, the multiple-point method and most of the two-point methods' load variations examined in this research provided an accurate (from large-moderate to perfect) estimate of the 1RM. Therefore, we recommend using the multi-point method and especially the two-point methods variations including higher relative loads to estimate 1RM.

\section{Introduction}

Continuous improvement of strength and power variables is required to increase athletic performance (Suchomel et al., 2018). Exercise intensity or load is generally acknowledged as the most important stimulus related to changes in strength levels and has been commonly identified as a percentage of maximum strength (Crewther et al., 2006; González-Badillo \& SánchezMedina, 2010). Maximal strength is related to improved force-time characteristics and general and sport-specific skill performance. Therefore, it plays an essential role in most sports (Wisløff et al., 2004). The one-repetition maximum (1RM) is widely used in practice and scientific research to determine training intensities and loads during the resistance training programs (Wood, Maddalozzo \& Harter, 2002; Kraemer \& Ratamess, 2004; Scott et al., 2016). The 1RM - can be determined either directly or indirectly, but the direct determination of the 1RM from one maximal lift has several disadvantages, such as an increase in the risk of injury if performed incorrectly or by inexperienced subjects (Balsalobre-Fernández et al., 2018; Ruf, Chéry \& Taylor, 2018). Besides, it is time-consuming and possibly impractical for large groups and requires fully motivated subjects to achieve their actual 1RM (Niewiadomski et al., 2008; Bazuelo-Ruiz et al., 2015). In addition, individual stress factors (e.g., sports stress, life stress, and social stress) cause fluctuations in the ability to move an external resistance (Fry \& Kraemer, 1997), and this can affect the daily stability of the 1RM level (Mann, Ivey \& Sayers, 2015). Therefore, considering the disadvantages of 1RM tests, researchers have developed various indirect prediction methodologies and regression equations to estimate 1RM using submaximal loads (Kravitz et al., 2003).

In recent years, among the indirect methodologies to estimate 1RM, the most widely used is the load-velocity (L-V) relationship (Jidovtseff et al., 2011; González-Badillo \& Sánchez-Medina, 2010). Several researchers have reported a highly linear relationship between movement velocity and relative load (\%1RM) in various resistance training exercises due to advancements in kinetic 
79

80

81

82

83

84

85

86

87

88

89

90

91

92

93

94

95

96

97

98

99

100

101

102

103

104

105

106

107

108

109

110

111

112

113

114

115

116

117

118

and kinematic transducer technologies that accurately measure bar velocity (González-Badillo \& Sánchez-Medina, 2010; Sánchez-Medina \& González-Badillo, 2011; Sánchez-Medina et al., 2014; Conceição et al., 2016). The L-V relationship equations have been used to predict the 1RM by using submaximal loads (Jidovtseff et al., 2011; Garcia-Ramos et al., 2018). The general load-velocity (L-V) relationships (Hecksteden et al., 2018) and the individual L-V relationships that use regression equations as the load associated with the mean velocity (MV) of the 1RM were proposed to estimate the 1RM (Weakley et al., 2021). Although the researchers reported a strong and approximately linear L-V relationship for the general method (GonzálezBadillo \& Sánchez-Medina, 2010; Sánchez-Medina \& González-Badillo, 2011; Conceição et al., 2016; Muñoz-López et al., 2017; García-Ramos et al., 2018), the affecting of MV and L-V relationship by the type (Sánchez-Medina \& González-Badillo, 2011; Helms et al., 2017) and implementation of exercises (García-Ramos et al., 2018; Pérez-Castilla et al., 2020) and sample groups (Torrejón et al., 2019) may limit its use in practice. As an alternative to generalized group equations, the multiple-point method applied to the individual L-V relationship was proposed for a more accurate estimate of the value of 1RM (Jovanović \& Flanagan, 2014). The individual load-velocity relationship consists of evaluating the velocity outputs of multiple submaximal loads ranging from 4 or 9 (Helms et al., 2017). The multipoint method also showed an accurate estimate of the 1RM value (Jidovtseff et al., 2011; Picerno et al., 2016; Helms et al., 2017; Pérez-Castilla et al., 2020), but multi-point-based individualized L-V protocols can often be time-consuming and prone to fatigue (García-Ramos et al., 2018).

Considering the limitations of the two methods mentioned above and assuming that the L-V relationship in resistance exercises is stable and linear (González-Badillo \& Sánchez-Medina, 2010; Bazuelo-Ruiz et al., 2015; Conceição et al., 2016), the 2-point method has been proposed by Jaric as an alternative (Jaric, 2016). The two-point method analyzes two different external loads to determine the L-V relationship instead of multiple loads (McBurnie et al., 2019). The 2point method has a quick and fatigue-free procedure to predict the 1RM (Weakley et al., 2021). The researchers recommend using two distant loads corresponding to approximately 45, 50\%$80,85 \%$ of 1RM in the protocol of the 2-point method (García-Ramos et al., 2018; PérezCastilla et al., 2021). However, the question of what should be the optimal 2 loads to be used in this method is still current (García-Ramos et al., 2018; Pérez-Castilla et al., 2018).

The validity of the 2-point method for upper-body free-weight exercises such as bench pull (García-Ramos et al., 2019), bench press (Jiménez-Alonso et al., 2020), lat pull-down, and seated cable row (Pérez-Castilla et al., 2021) has been confirmed. However, its validity for freeweight lower-body exercises (e.g., conventional deadlift, squat) has never been explored (Weakley et al., 2021). Regarding that, the researchers assume that the inconsistencies in the prediction accuracy of lower body exercises may be due to the technical complexity (Weakley et $a l$., 2021) of these movements. This hypothesis needs to be scientifically proven by standardizing the application techniques of the exercises during the research. For the reasons stated above, the aims of this study were (1) to examine the concurrent validity and reliability of the multi-point method and the two-point method's variations for estimating 1RM in the deadlift and squat 
119

120

121

122

123

124

125

126

127

128

129

130

131

132

133

134

135

136

137

138

139

140

141

142

143

144

145

146

147

148

149

150

151

152

153

154

155

156

157

158

exercises and (2) to identify the optimal combination of the two loads that allows predicting the $1 \mathrm{RM}$ with the highest accuracy.

\section{Materials \& Methods}

Study Design

In this study, we investigated the reliability and validity of the multiple-point method and twopoint method to predict 1RM for back squat and deadlift. The linear regression equation was computed using an individualized load-velocity relationship using mean velocity data of different submaximal loads $(\% 40,60,80,90)$. For the multiple-point method, each submaximal load and velocity were considered, meanwhile different two-point combinations (\%40-60, \%4080, \%40-90, \%60-80, \%60-90) were used to predict 1RM.

Investigations had been conducted for 6 days separated by 48 hours resting periods. On the first day and the fourth day, subjects' squat 1RM and the deadlift 1RM loads were determined and familiarizations were done for further velocity-based assessments. On the second, third, fifth and sixth day, 1RM tests were conducted while saving barbell velocity data to predict 1RM by using a linear regression equation.

\section{Participants}

Thirteen resistance-trained men participated in the study $(23.6 \pm 4.2 \mathrm{yrs}, 179.6 \pm 7.1 \mathrm{~m}, 80.2 \pm 8.9$

$\mathrm{kg}$ ). Including criteria were performing both exercises with a good pattern, at least 2 years of strength training experience. All subjects were free from any musculoskeletal injuries. Before the study, all subjects were informed of the purpose and risks of the investigation and provided signed informed consent. This study was approved by Yalova University Clinic Researches Ethics Committee (protocol number 2021/13, 11/03/2021) and was conducted in accordance with the Declaration of Helsinki.

\section{Procedures}

Athletes performed 6 testing sessions separated by 48 hours each other. Squat assessments were done on the first, the second, and the third day, whereas deadlift assessments were done on the fourth, the fifth, and the sixth day. On the first day, and the fourth day, familiarization sessions were conducted for each exercise. In these sessions, self-determined 1RM's relative loads were used to determine the actual 1RM score. Subjects began with a standardized warm-up followed by an incremental 1RM test. On the second and the third day for squat, on the fifth and the sixth day for deadlift subjects followed the same warm-up protocol and 1RM assessments. Relative loads that were used in the second, the third, the fifth, and the sixth day were determined from the initial 1RM assessment.

Regarding 1RM assessment, before each test, a standardized warm-up consisting of 8 minutes of jogging, 5 minutes of lower extremity mobility exercises, and 3 reps of the deadlift and the squat with $20 \mathrm{~kg}$ barbell was done. A custom-built power cage (Life Fitness, Hammer Strength, Rosemont, IL, USA) and $20 \mathrm{~kg}$ barbell (Eleiko, Halmstad, Sweden) were used for all 1RM assessments. In the first familiarization session, subjects were taught to perform squat at $90^{\circ}$ of the knee joint for the standardization of squat exercise. A string placed between two tripods, set 
159 for each individual to make them perform squat at $90^{\circ}$ was used for all squat assessments. Each 160 athlete touched the string with their hip in the eccentric phase of squat movement (Bazuelo-Ruiz 161 et al., 2015). When the eccentric phase of the squat was ended at $90^{\circ}$, subjects waited for 2

162

163

164

165

166

167

168

169

170

171

172

173

174

175

176

177

178

179

180

181

182

183

184

185

186

187

188

189

190

191

192

193

194

195

196

197

198

seconds to inactivate the stretch-shortening cycle and performed the concentric phase as fast as possible. On the fourth day (familiarization day of deadlift assessments), a technical model was shown and explained for deadlift exercise. Rounded lower or upper back and no full extension of the hip were taken into account as a failure (Ruf, Chéry \& Taylor, 2018). For the beginning and the finishing position of the deadlift, weight plates hat to touch the ground. When the eccentric phase of deadlift was ended, subjects waited for 2 seconds to inactivate the stretch-shortening cycle and performed the concentric phase as fast as possible. Verbal encouragement was given for each exercise. Incremental test protocol was adapted from previous research (Banyard, Nosaka \& Haff, 2017). For the familiarization sessions, subjects started with $\% 20$ loads of their self-determined 1 repetition maximum. They performed 3 reps at $\% 201 \mathrm{RM}, 3$ reps at $\% 40$ of $1 \mathrm{RM}, 3$ reps at $\% 60$ of $1 \mathrm{RM}, 1$ rep at $\% 80$ of $1 \mathrm{RM}$, and 1 rep at $\% 90$. The load was increased 2.5-5 kg after each successful attempt until the subject failure to lift. The rest period between sets was 3 minutes. For the velocity assessments, relative loads of 1RM that were determined in the $1 \mathrm{RM}$ test were used. Subjects performed the same warm-up and the same incremental test protocol.

Regarding velocity measurements, barbell velocity data was collected by means of a linear position transducer GymAware (GymAware Power Tool, Kinetic Performance Technologies, Canberra, Australia), whose validity was reported in previous studies (O'Donnell et al., 2018; Thompson et al., 2020). For each relative load, the highest mean velocity (MV) data was saved for statistical analysis. The device was connected to a tablet (iPad, Apple Inc., CA, USA) through bluetooth and barbell velocity data was recorded with the GymAware application (software version 1.2.1).

\section{Statistical Analyses}

All the statistical procedures were computed in an Excel spreadsheet designed by Hopkins (2017). Descriptive statistics were calculated for all variables and reported as mean \pm standard deviations (SD). The normality of the data was examined using the Kolmogorov-Smirnov test. It was determined that the data showed normal distribution. The level of the relationship between the measurements obtained with different load velocity profile methods was determined by means of the Pearson correlation test. The results of different methods using linear regression analysis were transformed into images using a correlation graph and $r$ values and confidence intervals were drawn on the graphs. One-way ANOVA test was used to examine the differences of estimates obtained by the different load-velocity methods. Inter-session reliability was expressed with coefficients of variation (CV) calculated from mean and standard deviations (standard error of measurement (typical error) / participants mean score $\times 100$ ). The uncertainty was expressed as $95 \%$ confidence interval (CI). Smallest worthwhile change (SWC) was calculated by multiplying the between-subject standard deviation (SD) of the performance by 0.2 (SWC $=0.2 \cdot$ between-subject SD [42], Lacome et al., 2019). The validity of different load velocity

Peer) reviewing PDF | (2021:10:66573:2:0:NEW 26 Jan 2022) 
199 estimation methods was tested using the Bland-Altman plot systematic deviation \pm random error.

200 The presence of heteroscedasticity was analyzed using correlation $\left(\mathrm{R}^{2}\right)$ between absolute

201 differences for validity.

202 As the level of the relationship, minor (0.0), small $(0.1)$, medium $(0.3)$, large $(0.5)$, very large

203 (0.7), almost perfect (0.9) and perfect (1.0) levels were used (Hopkins, 2017). The CV was rated

204 as good when $\mathrm{CV}<5 \%$, as moderate when $\mathrm{CV}$ was $5-10 \%$, and as poor when $\mathrm{CV}$ was $>10 \%$.

$205 \mathrm{p}<0.05$ alpha level was used as the significance level.

206

207

208

\section{Results}

209

Table 1 shows the difference between the estimates obtained by means of the different methods

210 in the deadlift exercise and the values obtained with the reference ("real") method. The inter-

211 session reliability of the methods used in the deadlift exercise in two separate sessions is shown

212 in Table 2. Table 3 shows the difference between the estimates obtained by different methods in

213 the squat exercise and the values obtained with the real method. The inter-session reliability of

214 Figures 1 and 2 show the differences of different methods from the real value. Figures 3 and 4 the methods used in the squat exercise in two separate sessions is shown in Table 4. 215 show the relationships of different methods with the real value. Bland-Altman plots showing the differences between data methods derived from the load velocity profile in exercises are shown

217 in Fig. 5 and 6. Both the multi-point method and the two-point method load variations showed

218 reliable results for $1 \mathrm{RM}$ estimation $(\mathrm{CV}<10 \%)$ squat and deadlift movements. Session-session

219

220 reliability was found to be low in deadlift (ICC: $0.171-0.335$ ) and squat exercises (ICC: $0.235-$ 0.479 ) of $40-60 \%$ and $60-80 \%$ in two-point methods. Deadlift (ICC: $0.815-0.996$ ) and squat

221

222 (ICC: $0.817-0.988$ ) had high session-to-session reliability in all other methods. Regarding the validity of deadlift exercise, the multipoint method $\left(\mathrm{R}^{2}=0.864\right)$ and 2 variations of two-point

223 method $\left(\mathrm{R}^{2}=0.816\right.$ for $40-80 \%, \mathrm{R}^{2}=0.732$ for $\left.60-80 \%\right)$ showed very large correlations, whereas

224 other 2 variations of the two-point method $\left(\mathrm{R}^{2}=0.945\right.$ for $40-90 \%, \mathrm{R}^{2}=0.914$ for $\left.60-90 \%\right)$ showed

225 almost perfect correlations with the actual 1RM. Regarding the validity of squat exercise, the

226

227 multi-point method $\left(\mathrm{R}^{2}=0.773\right)$ and 2 variations of the two-point method $\left(\mathrm{R}^{2}=0.0847\right.$ for 60 $80 \% \%, \mathrm{R}^{2}=0.705$ for $40-90 \%$ ) showed very large correlations, whereas $40-60 \%$ variation showed

228 almost perfect correlation $\left(\mathrm{R}^{2}=0.962\right)$ with the actual $1 \mathrm{RM}$.

229

230

231

232

\section{Discussion}

This study aimed at examining the concurrent validity and reliability of the multi-point method

233 and the two-point method for estimating 1RM in the deadlift and squat exercises and to

234 determine the accuracy of which optimal two loads variation can be used for the 2-point method

235 protocol. Our main findings revealed that both the multi-point method and the two-point method

236 variations showed reliable results for $1 \mathrm{RM}$ estimation $(\mathrm{CV}<10 \%)$ squat and deadlift exercises.

237 Session-session reliability was found to be low in deadlift (ICC: $0.171-0.335$ ) and squat exercises (ICC: $0.235-0.479$ ) of $40-60 \%$ and $60-80 \%$ in two-point methods. Deadlift (ICC: 
238

239

240

241

242

243

244

245

246

247

248

249

250

251

252

253

254

255

256

257

258

259

260

261

262

263

264

265

266

267

268

269

270

271

272

273

274

275

276

0.815-0.996) and squat (ICC: 0.817-0.988) had high session-to-session reliability in all other methods.

Regarding the validity of deadlift exercise, the multipoint method $\left(\mathrm{R}^{2}=0.864\right)$ and 2 variations of two-point method $\left(\mathrm{R}^{2}=0.816\right.$ for $40-80 \%, \mathrm{R}^{2}=0.732$ for $\left.60-80 \%\right)$ showed very large correlation, whereas other 2 variations of the two-point method $\left(R^{2}=0.945\right.$ for $40-90 \%, R^{2}=0.914$ for 60 $90 \%$ ) showed almost perfect correlation with the actual 1RM. Regarding the validity of squat exercise, the multi-point method $\left(\mathrm{R}^{2}=0.773\right)$ and 2 variations of the two-point method $\left(\mathrm{R}^{2}=0.0847\right.$ for $60-80 \% \%, \mathrm{R}^{2}=0.705$ for $\left.40-90 \%\right)$ showed very large correlation, whereas 40 $60 \%$ variation showed almost perfect correlation $\left(\mathrm{R}^{2}=0.962\right)$ with the actual $1 \mathrm{RM}$.

Blant-altman plots show that the differences between the real RM values of the multi-point and two-point method's variations (40-80\%, 40-90\%, and 60-80\%) in deadlift exercise are between acceptable upper and lower limits. However, it shows that the $40-60 \%$ and $60-90 \%$ variations are not between the acceptable upper and lower limits with real RM percentages. Details of the results are given in figure 5. For the squat exercise, Blant-altman plots show that the differences between the real RM values of the multi-point and two-point method's variations (40-80\%, 40$90 \%$, and $60-90 \%$ ) are between acceptable upper and lower limits. However, it shows that the $40-60 \%$ and $60-80 \%$ variations are not between the acceptable upper and lower limits with real RM percentages. Details of the results are given in figure 6.

Past studies that examined 1RM estimation methods using the L-V relationship reported different results for lower-body (squat, deadlift) and upper-body (bench press) compound exercises. For example, whereas the general validity results of studies on bench press exercise (GonzálezBadillo \& Sánchez-Medina, 2010; Jidovtseff et al., 2011; Sánchez-Medina et al., 2014; Loturco et al., 2017; García-Ramos et al., 2018) report a high correlation for L-V relationship and 1RM, the results for squat and deadlift exercises seem to be inconsistent (Sánchez-Medina \& GonzálezBadillo, 2011; Bazuelo-Ruiz et al., 2015; Banyard, Nosaka \& Haff, 2017; Lake et al., 2017). Researchers explain this result variation in lower-body exercises for several reasons. Although we attach importance to standardization practices among the measurements in the present study, we especially associate the differentiation of reliability and validity results with the following reasons. Weakley et al. (2021) attribute discrepancies in the accuracy of 1RM estimation to the greater technical complexity of squat and deadlift exercises compared with upper-body exercises. On the other hand, Balsalobre-Fernandes and Torres Ronda (2021) indicate that oscillations cause angle deviations up to $20^{\circ}$ in exercises that require vertical lifting such as squat. This situation may cause measurement errors in studies where free weight exercises with angular momentum components are examined with linear transducers, as in this study. Therefore, they stated that most of the studies analyzing the L-V relationship use the Smith Machine tool, which guarantees the vertical pathway of the barbell to avoid horizontal displacements. In fact, the researchers tried to use multiple linear position transducers instruments placed in four directions (positioned above and anterior, above and posterior) in order to avoid asymmetrical differences in the barbell in these exercises (Banyard, Nosaka \& Haff, 2017).

Peer) reviewing PDF | (2021:10:66573:2:0:NEW 26 Jan 2022) 
277 Past studies that examined the L-V relationships different methods in free weight lower body 278 exercises to estimate 1RM also reported variable results on validity and reliability. Banyard, 279 Nosaka and Haff (2017) found that the variations of the multiple-point method for back squat 280 exercise differ from 3 to 5 loads and they stated that the L-V relationship was moderately reliable 281 and valid, but could not accurately predict the 1RM. Yet, in extensive reviews written on 1RM 282 estimation methods, the authors concluded that the multi-point method can be time-consuming 283 and prone to fatigue (Garcia-Ramos \& Jaric, 2018; McBurnie et al., 2019). Although Ruf, Chéry 284 and Taylor (2018) reached reliable results in their research in which they examined various 285 variations of the two-point method in deadlift exercise, they reported that predicted 1RM scores 286 computed from all submaximal load ranges substantially overestimated the actual 1RM with 287 considerable differences between athletes. Lake et al. (2017) found more extreme results 288 compared with the studies highlighted above. Whereas they reported poor to moderate reliability, 289 they found that the estimated deadlift 1RMs were as much as $15 \%$ less than actual 1RMs. For the 290 estimation methods used in lower-body exercises that cannot accurately measure the actual 1RM, 291 the researchers also recommend acceptable kg errors. For example, Jukic et al. (2020) compared 2926 prediction methods, including the multi-point and two-point methods (40-90\%), and found that 293 no method for deadlift exercise could accurately predict the actual 1RM. That is why they have 294 suggested error limits of $5 \mathrm{~kg}$ to sports professionals. In addition to biomechanical and exercise 295 technique factors in lower body exercises, the fact that minimal velocity thresholds (MVT) are 296 exercise-specific should also be considered. Whereas researchers have found back squat MVT to 297 be around $0.30 \mathrm{~m} \cdot \mathrm{s}^{-1}$, others have recorded deadlift 1RM velocities as $0.16-0.17 \mathrm{~m} \cdot \mathrm{s}^{-1}$ (Izquierdo

298

299

300

301

302

303

304

305

306

307

308

309

310

311

312

313

314

315

316

\section{et al., 2006; Sanchez-Medina, Perez \& Gonzalez-Badillo, 2010). So that in the regression} analysis we used $0.30 \mathrm{~m} \cdot \mathrm{s}^{-1}$ for squat and $0.16 \mathrm{~m} \cdot \mathrm{s}^{-1}$ for deadlift as previous researches reported. The two-point method is more practical and requires less time than the multipoint method, but there is no strong scientific opinion about which 2 most appropriate loads should be preferred with this method. García-Ramos et al. (2018) recommended different external loads representing approximately $50 \%$ and $80 \%$ of self-reported $1 \mathrm{RM}$. Based on our experience during the measurements of this research, we recommend not using loads (multi-point method) below $40 \%$ for light loads due to velocity inconsistencies. The results of our research show that the other load, which is heavier, should be around $90 \%$. Although this situation negatively affects the advantages of the two-point method on the formation of fatigue, its validity decreases as this load rate decreases. According to the validity results of this study, it was determined that the validity values of the two-point method variations were higher than the multiple-point method. These results further strengthened the advantages of the two-point method, such as requiring less time and being more practical. However, the problem of which two loads would be most appropriate to estimate 1RM in the two-point method remains. In our study, two load variations with high validity in squat and deadlift exercises were found to be different. We think that this is because the velocity thresholds are exercise-specific. Whereas García-Ramos et al. (2018) suggest using two very different loads in this method, researchers also stated that heavy relative loads in the two-point method may cause problems in inexperienced athletes. Most of the variations with high validity in this study

Peerj reviewing PDF | (2021:10:66573:2:0:NEW 26 Jan 2022) 
317 contain loads of $80 \%$ or more. Therefore, we think that the experience and strength level of the 318 athlete can be an important criterion in the use of the two-point method.

319 To the best of our knowledge, this is the only study that examined the reliability and validity of 320 the multi-point and two-point 1RM estimation methods in both squat and deadlift exercises. The 321 two-point method has never been validated during lower-body exercises yet (Weakley et al., 322 2021). However, the results of this research show that valid results can be obtained in many 323 versions of the two-point method if the measurement and standardization problems in lower 324 body exercises are solved. Despite all these estimation methods, researchers state that direct 325 measurement of 1RM in lower body exercises is more reliable than $\mathrm{L}-\mathrm{V}$ relationship methods using submaximal loads (Garcia-Ramos et al., 2018).

\section{Conclusions}

330

In conclusion, whereas both the multiple-point method and two-point method loads' variations

331 showed reliable results, the multiple-point method and most of the two-point method load variations examined in this research provided (from large moderate to perfect) accurate estimate

332

333 of the 1RM. Therefore, we recommend using the multi-point method and especially two-point methods variations including higher relative loads in estimating 1RM. While the two-point method may overcome some of the potential limitations associated with the direct determination of 1RM strength, professionals should also be aware that direct determination of 1RM appears to be the most reliable method in training programs where daily 1RM level is not essential. Lastly, strength and conditioning practitioners and sports scientists should take into account that MV was used for this research and other velocity types like MPV and PV may give different results.

\section{Acknowledgments}

342

We thank all subjects, who participated in

the study.

343

344

\section{References}

345

Balsalobre-Fernández C, Marchante D, Muñoz-López M, Jiménez SL. 2018. Validity and

346 reliability of a novel iPhone app for the measurement of barbell velocity and 1RM on the benchpress exercise. Journal of Sports Sciences 36:64-70. DOI: 10.1080/02640414.2017.1280610. Balsalobre-Fernández C, Torres-Ronda L. 2021. The Implementation of Velocity-Based Training Paradigm for Team Sports: Framework, Technologies, Practical Recommendations and Challenges. Sports (Basel, Switzerland) 9:47 DOI: 10.3390/sports9040047. Predict the 1RM Back Squat. 2017. Journal of strength and conditioning research 31:18971904. DOI: 10.1519/JSC.0000000000001657.

355 Predicting Maximal Dynamic Strength From the Load-Velocity Relationship in Squat Exercise.

356 Journal of strength and conditioning research 29:1999-2005. DOI:

357 10.1519/JSC.0000000000000821. 
358 Conceição F, Fernandes J, Lewis M, Gonzaléz-Badillo JJ, Jimenéz-Reyes P. 2016. Movement

359

360

361

362

363

364

365

366

367

368

369

370

371

372

373

374

375

376

377

378

379

380

381

382

383

384

385

386

387

388

389

390

391

392

393

394

395

396

velocity as a measure of exercise intensity in three lower limb exercises. Journal of Sports Sciences 34:1099-1106. DOI: 10.1080/02640414.2015.1090010.

Crewther B, Keogh J, Cronin J, Cook C. 2006. Possible stimuli for strength and power adaptation: acute hormonal responses. Sports medicine (Auckland, N.Z.) 36:215-238. DOI: 10.2165/00007256-200636030-00004.

Fry AC, Kraemer WJ. 1997. Resistance exercise overtraining and overreaching. Neuroendocrine responses. Sports medicine (Auckland, N.Z.) 23:106-129. DOI: 10.2165/00007256-19972302000004.

García-Ramos A, Barboza-González P, Ulloa-Díaz D, Rodriguez-Perea A, Martinez-Garcia D, Guede-Rojas F, Hinojosa-Riveros H, Chirosa-Ríos LJ, Cuevas-Aburto J, Janicijevic D, Weakley J. 2019. Reliability and validity of different methods of estimating the one-repetition maximum during the free-weight prone bench pull exercise. Journal of Sports Sciences 37:2205-2212. DOI: 10.1080/02640414.2019.1626071.

García-Ramos A, Haff GG, Pestaña-Melero FL, Pérez-Castilla A, Rojas FJ, BalsalobreFernández C, Jaric S. 2018. Feasibility of the 2-Point Method for Determining the 1-Repetition Maximum in the Bench Press Exercise. International journal of sports physiology and performance 13:474-481. DOI: 10.1123/ijspp.2017-0374.

Garcia-Ramos A, Jaric S. 2018. Two-point method: A quick and fatigue-free procedure for assessment of muscle mechanical capacities and the 1 repetition maximum. Strength \& Conditioning Journal 40:54-66. DOI: 10.1519/SSC.0000000000000359.

García-Ramos A, Pestaña-Melero FL, Pérez-Castilla A, Rojas FJ, Haff GG. 2018. Differences in the Load-Velocity Profile Between 4 Bench-Press Variants. International journal of sports physiology and performance 13:326-331. DOI: 10.1123/ijspp.2017-0158.

González-Badillo JJ, Sánchez-Medina L. 2010. Movement velocity as a measure of loading intensity in resistance training. International journal of sports medicine 31:347-352. DOI: 10.1055/s-0030-1248333.

Hecksteden A, Pitsch W, Rosenberger F, Meyer T. 2018. Repeated testing for the assessment of individual response to exercise training. Journal of applied physiology (Bethesda, Md. : 1985) 124:1567-1579. DOI: 10.1152/japplphysiol.00896.2017.

Helms ER, Storey A, Cross MR, Brown SR, Lenetsky S, Ramsay H, Dillen C, Zourdos MC. 2017. RPE and Velocity Relationships for the Back Squat, Bench Press, and Deadlift in Powerlifters. Journal of strength and conditioning research 31(2):292-297. DOI: 10.1519/JSC.0000000000001517.

Hopkins WG. 2017. Spreadsheets for analysis of validity and reliability. Sportscience 21. Izquierdo M, González-Badillo JJ, Häkkinen K, Ibáñez J, Kraemer WJ, Altadill A, Eslava J, Gorostiaga EM. 2006. Effect of loading on unintentional lifting velocity declines during single sets of repetitions to failure during upper and lower extremity muscle actions. International journal of sports medicine 27:718-724. DOI: 10.1055/s-2005-872825.

Peer) reviewing PDF | (2021:10:66573:2:0:NEW 26 Jan 2022) 
397 Kraemer WJ, Ratamess NA. 2004. Fundamentals of resistance training: progression and exercise

398

399

400

401

402

403

404

405

406

407

408

409

410

411

412

413

414

415

416

417

418

419

420

421

422

423

424

425

426

427

428

429

430

431

432

433

434

prescription. Medicine and science in sports and exercise 36:674-688. DOI:

10.1249/01.mss.0000121945.36635.61.

Kravitz L, Akalan C, Nowicki K, Kinzey SJ. 2003. Prediction of 1 repetition maximum in highschool power lifters. Journal of strength and conditioning research 17:167-172. DOI:

10.1519/1533-4287(2003)017<0167:pormih>2.0.co;2.

Jaric S. 2016. Two-Load Method for Distinguishing Between Muscle Force, Velocity, and

Power-Producing Capacities. Sports medicine (Auckland, N.Z.) 46(11):1585-1589. DOI: 10.1007/s40279-016-0531-z.

Jidovtseff B, Harris NK, Crielaard JM, Cronin JB. 2011. Using the load-velocity relationship for 1RM prediction. Journal of strength and conditioning research 25:267-270. DOI:

10.1519/JSC.0b013e3181b62c5f.

Jiménez-Alonso A, García-Ramos A, Cepero M, Miras-Moreno S, Rojas FJ, Pérez-Castilla A. 2020. Velocity Performance Feedback During the Free-Weight Bench Press Testing Procedure: An Effective Strategy to Increase the Reliability and One Repetition Maximum Accuracy

Prediction. Journal of strength and conditioning research. Epub ahead of print. DOI: 10.1519/JSC.0000000000003609.

Jovanović M, Flanagan EP. 2014. Researched applications of velocity based strength training. Journal of Australian Strength and Conditioning 22:58-69.

Jukic I, García-Ramos A, Malecek J, Omcirk D, Tufano JJ. 2020. Validity of Load-Velocity Relationship to Predict 1 Repetition Maximum During Deadlifts Performed With and Without Lifting Straps: The Accuracy of Six Prediction Models. Journal of strength and conditioning research. DOI: 10.1519/JSC.0000000000003596. Epub ahead of print.

Lacome M, Peeters A, Mathieu B, Bruno M, Christopher C, Piscione J. 2019. Can we use GPS for assessing sprinting performance in rugby sevens? A concurrent validity and between-device reliability study. Biology of sport 36:25-29. DOI: 10.5114/biolsport.2018.78903.

Lake J, Naworynsky D, Duncan F, Jackson M. 2017. Comparison of Different Minimal Velocity Thresholds to Establish Deadlift One Repetition Maximum. Sports (Basel, Switzerland) 5:70. DOI: $10.3390 /$ sports5030070.

Loturco I, Kobal R, Moraes JE, Kitamura K, Cal Abad CC, Pereira LA, Nakamura FY. 2017. Predicting the Maximum Dynamic Strength in Bench Press: The High Precision of the Bar Velocity Approach. Journal of strength and conditioning research 31:1127-1131. DOI: 10.1519/JSC.0000000000001670.

Mann JB, Ivey PA, Sayers SP. 2015. Velocity-based training in football. Strength \& Conditioning Journal 37:52-57. DOI: 10.1519/SSC.0000000000000177.

McBurnie AJ, Allen KP, Garry M, Martin M, Jones PA, Comfort P, McMahon JJ. 2019. The benefits and limitations of predicting one repetition maximum using the load-velocity relationship. Strength \& Conditioning Journal 41:28-40. DOI: 10.1519/SSC.0000000000000496.

Peer) reviewing PDF | (2021:10:66573:2:0:NEW 26 Jan 2022) 
435 Muñoz-López M, Marchante D, Cano-Ruiz MA, Chicharro JL, Balsalobre-Fernández C. 2017. 436 Load-, Force-, and Power-Velocity Relationships in the Prone Pull-Up Exercise. International 437 journal of sports physiology and performance 12:1249-1255. DOI: 10.1123/ijspp.2016-0657. 438 Niewiadomski W, Laskowska D, Gąsiorowska A, Cybulski G, Strasz A, Langfort J. 2008. 439 Determination and prediction of one repetition maximum (1RM): Safety considerations. Journal 440 of Human Kinetics 19:109-120. DOI: 10.2478/v10078-008-0008-8.

441 O’Donnell S, Tavares F, McMaster D, Chambers S, Driller M. 2018. The validity and reliability 442 of the GymAwxare linear position transducer for measuring counter-movement jump 443 performance in female athletes. Measurement in Physical Education and Exercise Science 444 22:101-107. DOI: 10.1080/1091367X.2017.1399892.

445 Pérez-Castilla A, García-Ramos A, Padial P, Morales-Artacho AJ, Feriche B. 2020. Load446 Velocity Relationship in Variations of the Half-Squat Exercise: Influence of Execution 447 Technique. Journal of strength and conditioning research 34:1024-1031. DOI:

448 10.1519/JSC.0000000000002072.

449 Pérez-Castilla A, Suzovic D, Domanovic A, Fernandes JFT, García-Ramos A. (2021). Validity 450 of Different Velocity-Based Methods and Repetitions-to-Failure Equations for Predicting the 1 451 Repetition Maximum During 2 Upper-Body Pulling Exercises. Journal of strength and 452 conditioning research 35:1800-1808. DOI: 10.1519/JSC.0000000000003076.

453 Picerno P, Iannetta D, Comotto S, Donati M, Pecoraro F, Zok M, Tollis G, Figura M, Varalda C, 454 Di Muzio D, Patrizio F, Piacentini MF. 2016. 1RM prediction: a novel methodology based on 455 the force-velocity and load-velocity relationships. European journal of applied physiology 456 116:2035-2043. DOI: 10.1007/s00421-016-3457-0.

457 Sánchez-Medina L, González-Badillo JJ. 2011. Velocity loss as an indicator of neuromuscular 458 fatigue during resistance training. Medicine and science in sports and exercise 43:1725-1734. 459 DOI: 10.1249/MSS.0b013e318213f880.

460 Sánchez-Medina L, González-Badillo JJ, Pérez CE, Pallarés JG. 2014. Velocity- and power-load 461 relationships of the bench pull vs. bench press exercises. International journal of sports medicine 462 35:209-216. DOI: 10.1055/s-0033-1351252.

463 Sanchez-Medina L, Perez CE, Gonzalez-Badillo JJ. 2010. Importance of the propulsive phase in 464 strength assessment. International journal of sports medicine 31:123-129. DOI: 10.1055/s-00294651242815.

466 Scott BR, Duthie GM, Thornton HR, Dascombe BJ. 2016. Training Monitoring for Resistance 467 Exercise: Theory and Applications. Sports medicine (Auckland, N.Z.) 46:687-698. DOI:

468 10.1007/s40279-015-0454-0.

469 Ruf L, Chéry C, Taylor KL. 2018. Validity and Reliability of the Load-Velocity Relationship to 470 Predict the One-Repetition Maximum in Deadlift. Journal of strength and conditioning research 471 32:681-689. DOI: 10.1519/JSC.0000000000002369.

472 Suchomel TJ, Nimphius S, Bellon CR, Stone MH. 2018. The Importance of Muscular Strength: 473 Training Considerations. Sports medicine (Auckland, N.Z.) 48:765-785. DOI: 10.1007/s40279474 018-0862-z. 
475 Thompson SW, Rogerson D, Dorrell HF, Ruddock A, Barnes A. 2020. The Reliability and 476 Validity of Current Technologies for Measuring Barbell Velocity in the Free-Weight Back Squat 477 and Power Clean. Sports (Basel, Switzerland) 8:94. DOI: 10.3390/sports8070094.

478 Torrejón A, Balsalobre-Fernández C, Haff GG, García-Ramos A. 2019. The load-velocity profile 479 differs more between men and women than between individuals with different strength levels.

480 Sports biomechanics 18:245-255. DOI: 10.1080/14763141.2018.1433872.

481 Weakley J, Mann B, Banyard H, McLaren S, Scott T, Garcia-Ramos A. 2021. Velocity-based 482 training: From theory to application. Strength \& Conditioning Journal 43:31-49. DOI:

$48310.1519 /$ SSC.0000000000000560.

484 Wisløff U, Castagna C, Helgerud J, Jones R, Hoff J. 2004. Strong correlation of maximal squat 485 strength with sprint performance and vertical jump height in elite soccer players. British journal 486 of sports medicine 38:285-288. DOI: 10.1136/bjsm.2002.002071.

487 Wood TM, Maddalozzo GF, Harter RA. 2002. Accuracy of seven equations for predicting 1-RM 488 performance of apparently healthy, sedentary older adults. Measurement in physical education 489 and exercise science 6:67-94. DOI: 10.1207/S15327841MPEE0602_1. 
Figure 1

Differences between methods of data derived from the load velocity profile in deadlift exercise. 


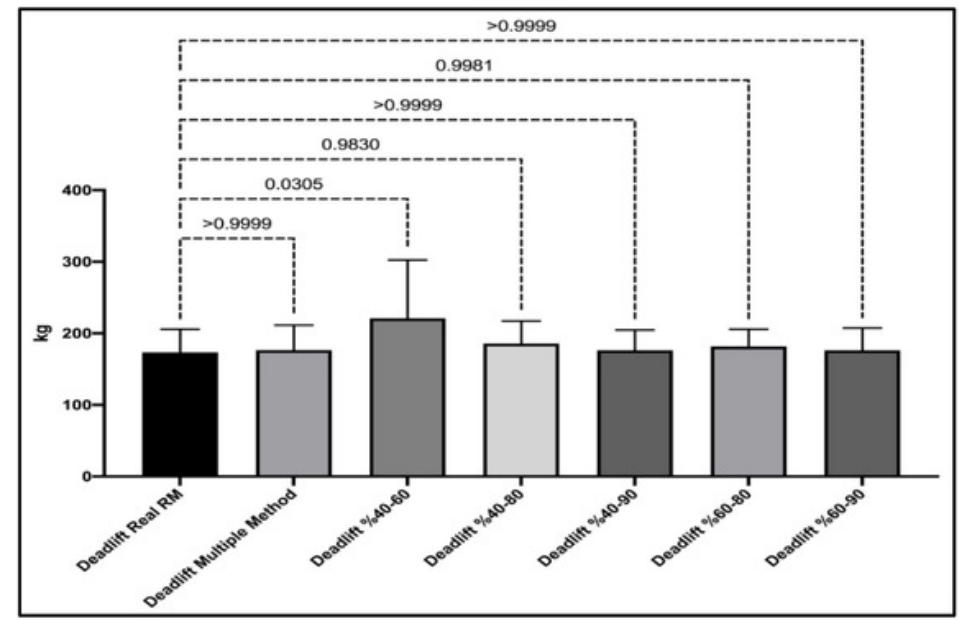

Figure 1. Differences between methods of data derived from the load velocity profile in deadlift exercise. 
Figure 2

Differences between methods of data derived from the load velocity profile in squat exercise. 


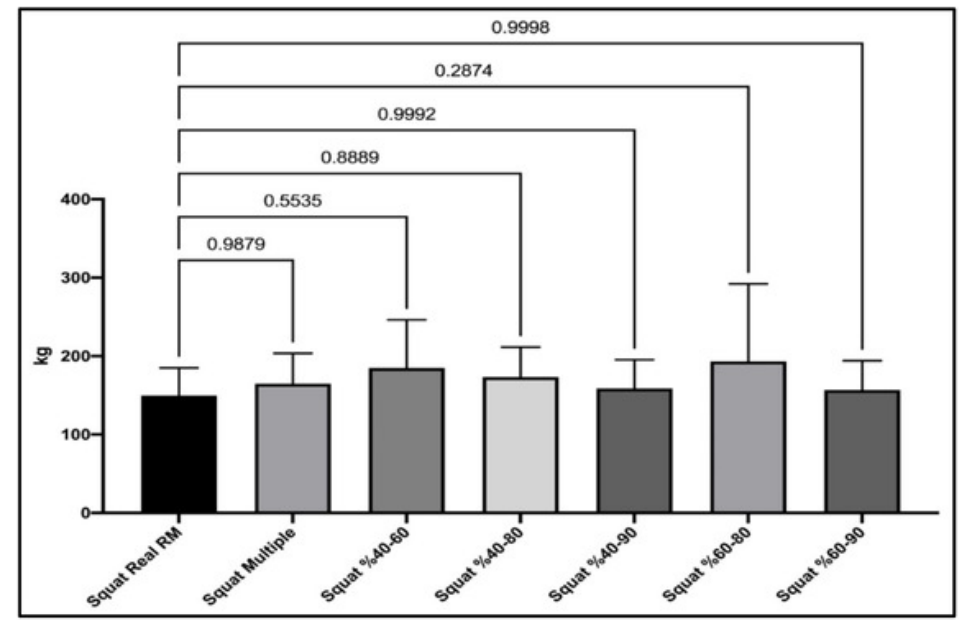

Figure 2. Differences between methods of data derived from the load velocity profile in squat exercise. 
Figure 3

Relationships of data derived from the load velocity profile in deadlift exercise between methods.
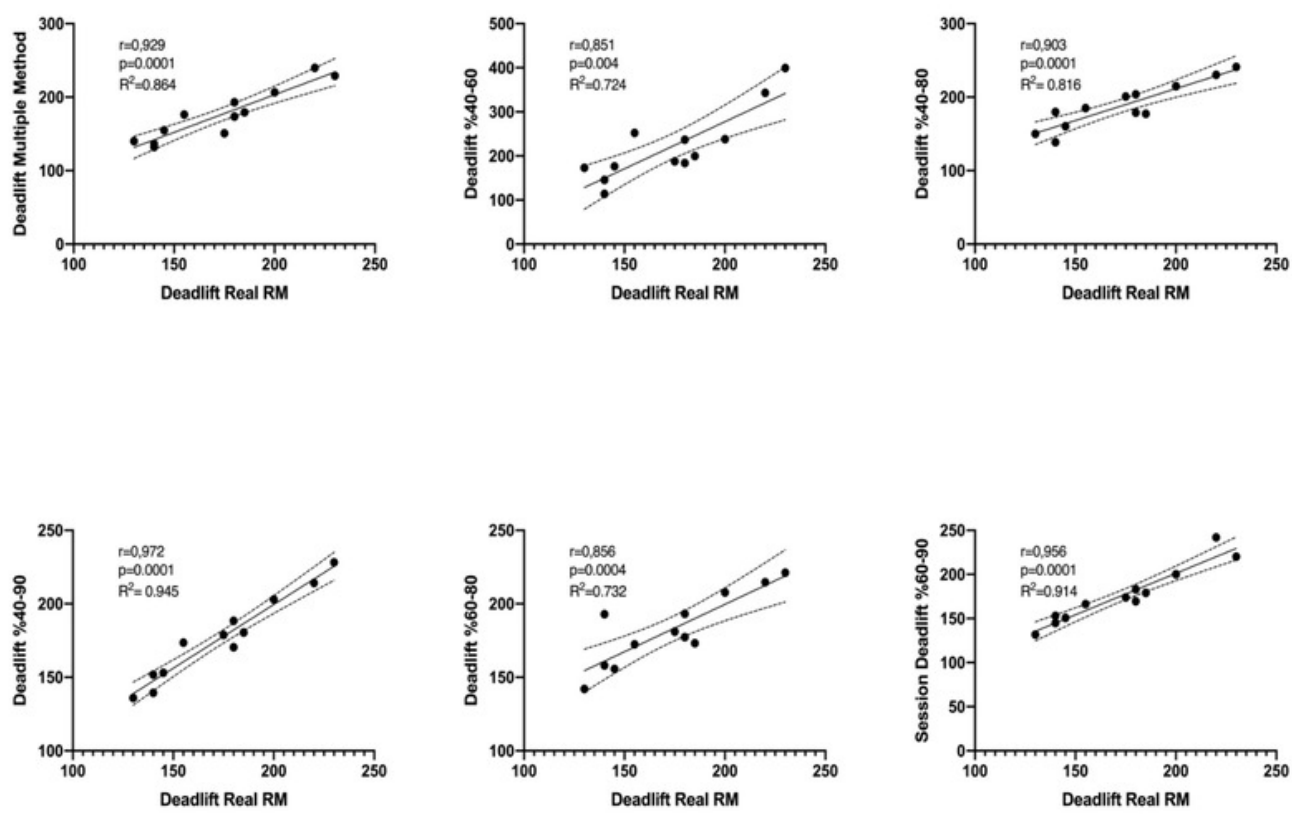

Figure 3. Relationships of data derived from the load velocity profile in deadlift exercise between methods. 
Figure 4

Relationships of data derived from the load velocity profile in squat exercise between methods.
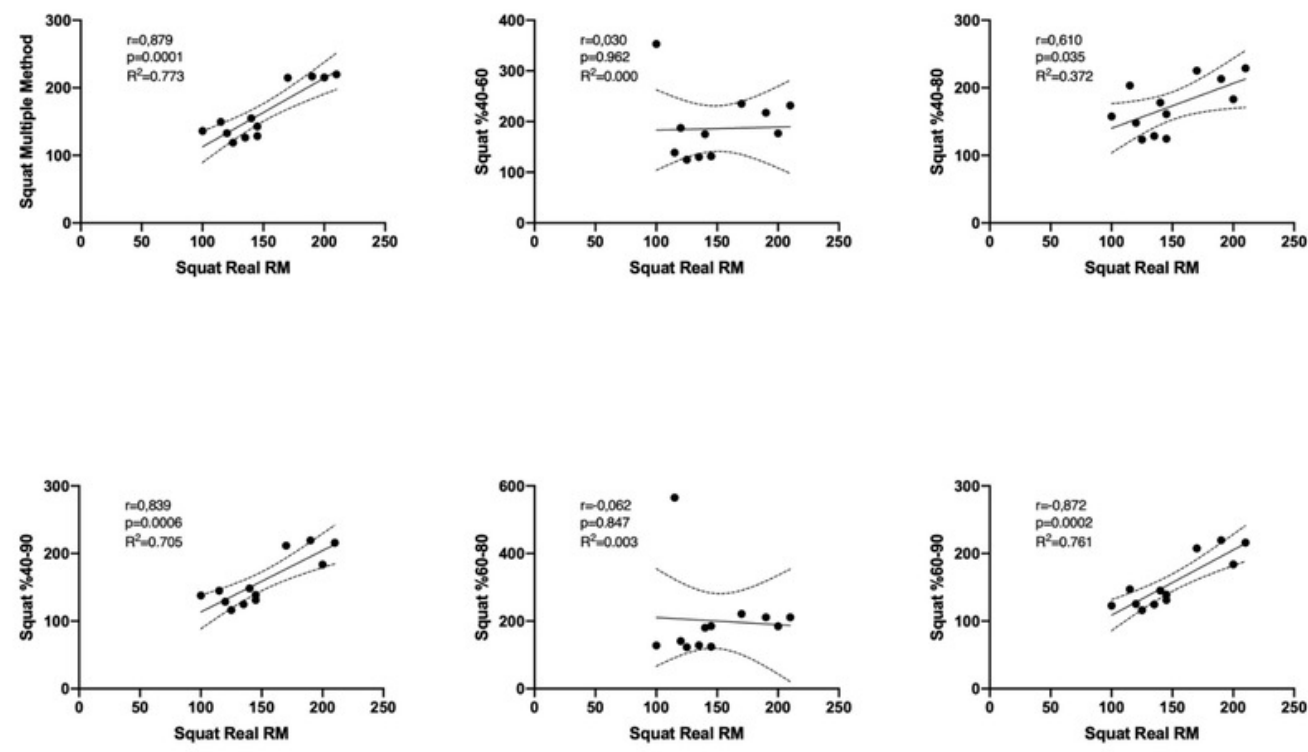

Figure 4. Relationships of data derived from the load velocity profile in squat exercise between methods. 
Figure 5

Bland-Altman plot showing the differences between methods of data derived from the load velocity profile in deadlift exercise. 

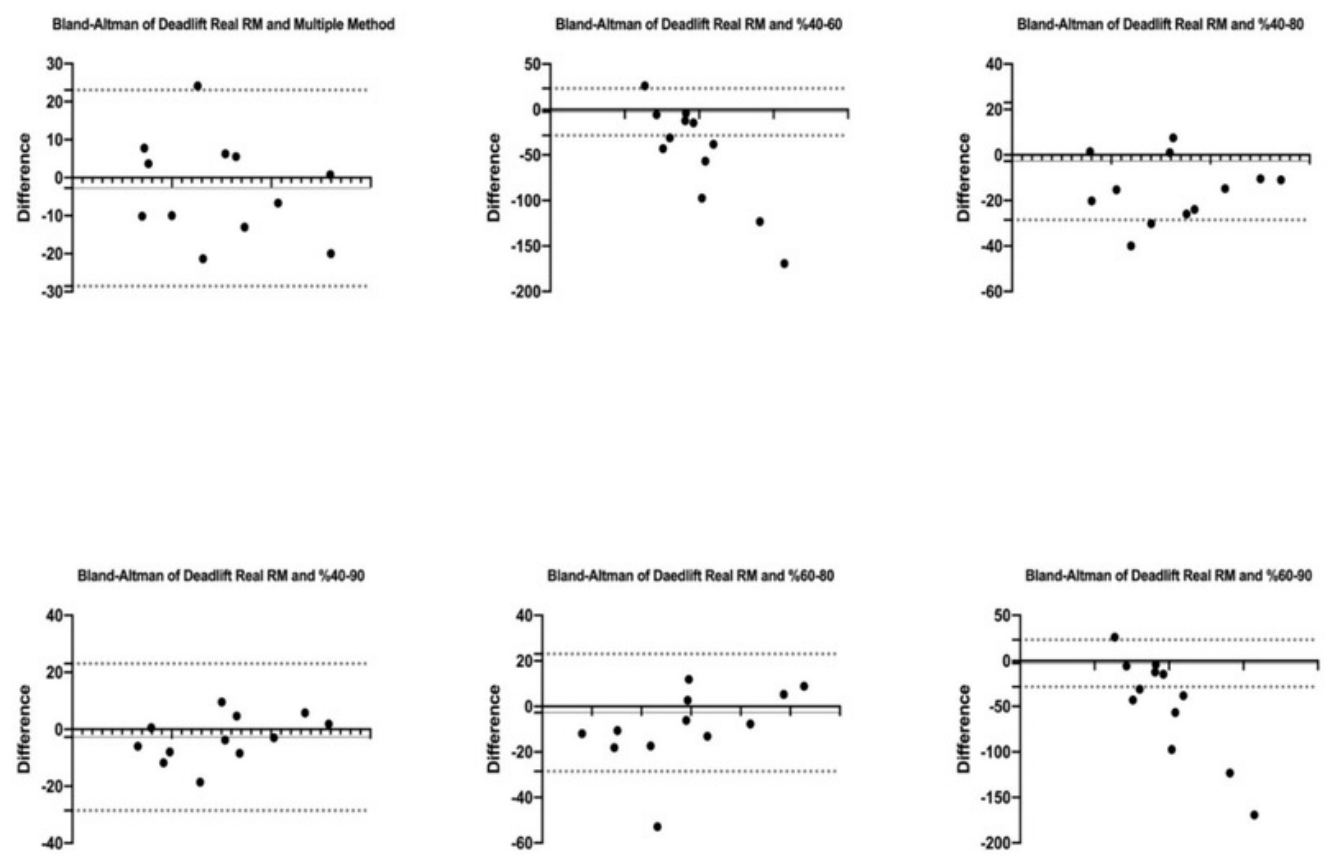

Figure 5. Bland-Altman plot showing the differences between methods of data derived from the load velocity profile in deadlift exercise. 
Figure 6

Bland-Altman plot showing the differences between methods of data derived from the load velocity profile in squat exercise.
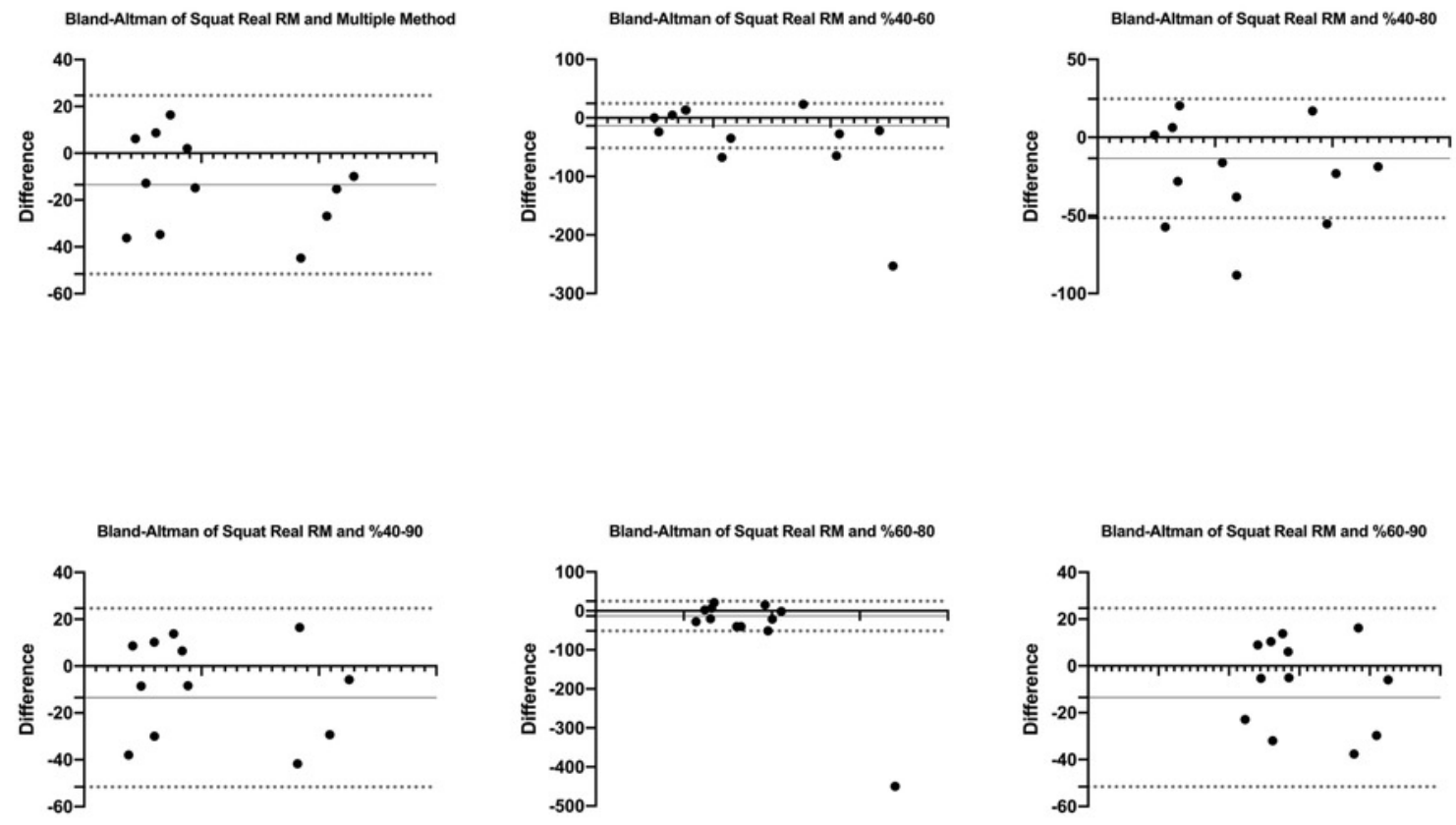

Figure 6. Bland-Altman plot showing the differences between methods of data derived from the load velocity profile in squat exercise. 


\section{Table 1 (on next page)}

Differences between methods of data derived from the load velocity profile in deadlift exercise.

$*_{p}<0.05$ 
1

Table 1. Differences between methods of data derived from the load velocity profile in deadlift exercise.

\begin{tabular}{|l|l|l|l|c|}
\hline Tukey's multiple comparisons test & $\begin{array}{l}\text { Mean } \\
\text { Difference }\end{array}$ & $\begin{array}{l}\mathbf{9 5 . 0 0 \%} \text { CI of } \\
\text { difference }\end{array}$ & p Value & $\begin{array}{c}\text { Mean } \\
\text { Absolute } \\
\text { Error }\end{array}$ \\
\hline Deadlift Real RM vs. Deadlift Multiple Method & -3.16 & -48.13 to 41.81 & $>0.9999$ & 7.057 \\
\hline Deadlift Real RM vs. Deadlift \%40-60 & -47.59 & -92.56 to -2.62 & $0.0305 *$ & 52.583 \\
\hline Deadlift Real RM vs. Deadlift \%40-80 & -12.28 & -57.24 to 32.69 & 0.9830 & 12.911 \\
\hline Deadlift Real RM vs. Deadlift \%40-90 & -2.995 & -47.96 to 41.97 & $>0.9999$ & 5.556 \\
\hline Deadlift Real RM vs. Deadlift \%60-80 & -8.23 & -53.20 to 36.74 & 0.9981 & 11.775 \\
\hline Deadlift Real RM vs. Deadlift \%60-90 & -2.97 & -47.93 to 42.00 & $>0.9999$ & 6.78 \\
\hline
\end{tabular}




\section{Table 2 (on next page)}

Inter-session reliability of the data obtained from the load-velocity profile in the deadlift exercise. 
1 Table 2. Inter-session reliability of the data obtained from the load-velocity profile in the deadlift exercise.

\begin{tabular}{|l|l|l|l|}
\hline Measurement Methods & CV\% & SWC & ICC \\
\hline Deadlift Multiple Method & 4,93 & 4.73 & 0.996 \\
\hline Deadlift \%40-60 & 7,72 & 7.38 & 0.171 \\
\hline Deadlift \%40-80 & 4,23 & 3.40 & 0.815 \\
\hline Deadlift \%40-90 & 4,27 & 3.20 & 0.996 \\
\hline Deadlift \%60-80 & 3,86 & 2.65 & 0.335 \\
\hline Deadlift \%60-90 & 4,62 & 3.53 & 0.972 \\
\hline
\end{tabular}

2 


\section{Table 3(on next page)}

Differences between methods of data derived from the load velocity profile in squat exercise. 
Table 3. Differences between methods of data derived from the load velocity profile in squat exercise.

\begin{tabular}{|l|l|l|l|c|}
\hline Tukey's multiple comparisons test & $\begin{array}{l}\text { Mean } \\
\text { Difference }\end{array}$ & $\begin{array}{l}\mathbf{9 5 , 0 0 \%} \text { CI of } \\
\text { difference }\end{array}$ & p Value & $\begin{array}{c}\text { Mean } \\
\text { Absolute } \\
\text { Error }\end{array}$ \\
\hline Squat Real RM vs. Squat Multiple & -14.95 & -73.43 to 43.53 & 0.9879 & 20.211 \\
\hline Squat Real RM vs. Squat \%40-60 & -35.12 & -93.60 to 23.36 & 0.5535 & 47.075 \\
\hline Squat Real RM vs. Squat \%40-80 & -23.70 & -82.18 to 34.78 & 0.8889 & 33.335 \\
\hline Squat Real RM vs. Squat \%40-90 & -9.223 & -67.70 to 49.26 & 0.9992 & 18.668 \\
\hline Squat Real RM vs. Squat \%60-80 & -43.58 & -102.1 to 14.90 & 0.2874 & 61.294 \\
\hline Squat Real RM vs. Squat \%60-90 & -7.31 & -65.79 to 51.17 & 0.9998 & 16.693 \\
\hline
\end{tabular}

2 


\section{Table 4 (on next page)}

Inter-session reliability of the data obtained from the load-velocity profile in the squat exercise. 
1 Table 4. Inter-session reliability of the data obtained from the load-velocity profile in the squat exercise.

\begin{tabular}{|l|l|l|c|}
\hline Measurement Methods & CV\% & SWC & ICC \\
\hline Squat Multiple Method & 4,42 & 4.72 & 0.817 \\
\hline Squat \%40-60 & 6,26 & 6.66 & 0.235 \\
\hline Squat \%40-80 & 4,07 & 4.40 & 0.822 \\
\hline Squat \%40-90 & 4,34 & 4.60 & 0.905 \\
\hline Squat \%60-80 & 9,81 & 10.25 & 0.479 \\
\hline Squat \%60-90 & 4,50 & 4.74 & 0.988 \\
\hline
\end{tabular}

2 